Article

\title{
Preparation of Few-Layered Wide Bandgap MoS2 with Nanometer Lateral Dimensions by Applying Laser Irradiation
}

\author{
Mitra Mahdavi ${ }^{1}$, Salimeh Kimiagar ${ }^{2, *(D)}$ and Fahimeh Abrinaei ${ }^{3}$ (D) \\ 1 Department of Physics, Central Tehran Branch, Islamic Azad University, Tehran 1584743311, Iran; \\ Mitramahdavi77@gmail.com \\ 2 Nano Research Lab (NRL), Department of Physics, Central Tehran Branch, Islamic Azad University, \\ Tehran 1584743311, Iran \\ 3 Department of Physics, East Tehran Branch, Islamic Azad University, Tehran 1584743311, Iran; \\ f_abrinaey@yahoo.com \\ * Correspondence: skimiagar@iauctb.ac.ir; Tel.: +98-9121230921; Fax: +98-2188074907
}

Received: 29 January 2020; Accepted: 20 February 2020; Published: 2 March 2020

\begin{abstract}
In this study, we report a new method for the quick, green, and one-step preparation of few-layered molybdenum disulfide $\left(\mathrm{MoS}_{2}\right)$ nanosheets with wide bandgap. $\mathrm{MoS}_{2}$ nanosheets with small lateral dimension and uniform size distribution were synthesized for various applications. $\mathrm{MoS}_{2}$ powder was synthesized using the hydrothermal method; then, thinned by applying laser irradiation with different energies from 40 to $80 \mathrm{~mJ}$. Transmission electron microscopy (TEM), X-ray diffraction (XRD), Fourier transform infrared spectroscopy (FTIR), UV-Vis absorption spectra, and photoluminescence (PL) spectra were applied for the characterization of the $\mathrm{MoS}_{2}$ nanosheets in terms of morphology, crystal structures, and optical properties. The widest calculated bandgap $4.7 \mathrm{eV}$ was for the sample under $80 \mathrm{~mJ}$ laser energy. The results confirmed the successful preparation of highly pure, uniform, and few-layered $\mathrm{MoS}_{2}$ nanosheets. Furthermore, it was possible to enhance the production rate of $\mathrm{MoS}_{2}$ nanosheets (including nanosheets and nanoparticles) through laser irradiation. Thus, the present paper introduces a simple and green alternative approach for preparing few-layered $\mathrm{MoS}_{2}$ nanosheets of transition metal dichalcogenides or other layered materials.
\end{abstract}

Keywords: few-layered; molybdenum disulfide; laser; low lateral dimension

\section{Introduction}

Lately, few-layered materials along with two-dimensional materials have attracted the most attention due to their diverse properties and applications [1-3]. These materials exhibit completely different properties as compared with their bulk counterparts making them one of the best candidates for new generations of electronic and optoelectronic devices [4-6]. The application of few-layered materials has contributed to advances in the fabrication of ultrathin layered materials with high surface-to-volume ratio [7-9]. Transition metal dichalcogenides (TMDs) are in a form of $\mathrm{MX}_{2}$, where $\mathrm{M}$ and $\mathrm{X}$ denote transition metal and chalcogen atoms, respectively. The TMDs are important two-dimensional (2D) materials, which include about 60 members. The structures of these layered materials are hexagonally packed. Due to this property, the TMDs, such as $\mathrm{MoS}_{2}, \mathrm{WS}_{2}, \mathrm{WTe}_{2}, \mathrm{TiS}_{2}, \mathrm{TaS}_{2}, \mathrm{ZrS} 2$, or graphite are exfoliated to prepare the 2D materials. Unlike graphene which has no bandgap, the TMDs are natural semiconductors. Therefore, they are applied in electronic devices and optical devices. The mobility of 2D TMDs is much lower than graphene, which limits their applications in devices. Zhang's group prepared few-layer black phosphorus and characterized a few-layer black phosphorus field-effect transistor [10]. A wafer-scale $\mathrm{MoS}_{2}$ was prepared by Yu et al. [11]. Novoselov et al. predicted some 
materials including boron nitride which could be exfoliated into a single layer [12]. Coleman et al. sonicated commercial molybdenum disulfide $\left(\mathrm{MoS}_{2}\right)$ and WS2 [13]. May et al. tried several polymers as stabilizers to exfoliate and disperse $\mathrm{MoS}_{2}$ and boron nitride [14]. Varrla et al. exfoliated molybdenum disulfide in aqueous surfactant solutions under shearing by a kitchen blender [15]. Zhang's group prepared $\mathrm{MoS}_{2}$ /polyvinylpyrrolidone (PVP) nanocomposites by sonication of $\mathrm{MoS}_{2}$ powder in ethanol with PVP [16]. So far, the practical application of $\mathrm{g}-\mathrm{CN}$ has been limited to photocatalytic water splitting, photodegradation of dyes, bioimaging, photocatalyst, and fluorescence applications [17-19].

Molybdenum disulfide $\left(\mathrm{MoS}_{2}\right)$ and tungsten disulfide $\left(\mathrm{WS}_{2}\right)$ are layered materials that show great potential to extend to few-layered sheets paving the way for the new physical phenomena $[20,21]$. $\mathrm{MoS}_{2}$ has been favored greatly due to its inherent structural characteristics that are composed of strong covalently S-Mo-S sheets detained by van der Waals interactions among the layered structures [22]. These weak interlayer interactions make the reduction of $\mathrm{MoS}_{2}$ layers possible by micromechanical exfoliation from bulk crystalline [23]. It is known that electronic properties, including the energy gap of $\mathrm{MoS}_{2}$, are strongly related to the number of atomic layers. Researches have shown that few-layered $\mathrm{MoS}_{2}$ turns into a direct bandgap semiconductor if thinned, while bulk $\mathrm{MoS}_{2}$ has an indirect bandgap, which is favorable for optoelectronics [24-26]. Due to this distinctive structure and features, $\mathrm{MoS}_{2}$ can be used in numerous fields such as biomedicine, energy storage, gas sensing, catalysis, and electronics engineering [27]. $\mathrm{MoS}_{2}$ has become an efficient electrocatalyst for hydrogen evolution reactions due to its abundant active edge sites, high specific surface area, and excellent electrical conductivity, together with strong hydrogen adsorption properties [28,29]. The small size particles show strong optical nonlinearity features [30]. $\mathrm{MoS}_{2}$ in both bulk and few-layered form has been applied in photovoltaics [31,32] and photocatalysis [33] applications as they are capable of efficiently absorbing a broad region of solar spectrum. Furthermore, more stable quantum size confinement effects are expected because of the even size distribution of the monolayer nanosheets. The quantum confinements in layered d-electron materials such as $\mathrm{MoS}_{2}$ pave the way to engineer the electronic structure of matter at the nanoscale. Consequently, finding an effective method to prepare uniform-size monolayer $\mathrm{MoS}_{2}$ nanosheets seems essential. The literature review shows that the current research on two-dimension (2D) $\mathrm{MoS}_{2}$ nanosheets is principally based on the exfoliation technique, chemical vapor deposition method, or solvothermal route [34-36]. The chemical vapor deposition method requires laborious experimental conditions including high temperature, high vacuum, and special substrates that probably would restrict the application of $2 \mathrm{D} \mathrm{MoS}_{2}$ nanosheets in practice. Simple and low-cost methods are needed for large-scale fabrication and commercial application of $\mathrm{MoS}_{2}$-based devices.

In this study, we report a new method for the quick, green, and one-step preparation of even monolayer $\mathrm{MoS}_{2}$ nanosheets. First $\mathrm{MoS}_{2}$ powder was synthesized using the hydrothermal method, and then they were thinned by reduction layers using laser irradiation. It is the first time to apply this method to exfoliate the $\mathrm{MoS}_{2}$ sheets. Next, we study the characteristic of $\mathrm{MoS}_{2}$ nanosheets.

\section{Experimental Details}

Materials for these experiments were acquired from Merck Company. To synthesize $\mathrm{MoS}_{2}$ powder, a solution of $0.1 \mathrm{M}$ ammonium heptamolybdate $\left(\mathrm{NH}_{4}\right)_{6} \mathrm{Mo}_{7} \mathrm{O}_{24}$ and $0.2 \mathrm{M}$ thiourea $\mathrm{CH}_{4} \mathrm{~N}_{2} \mathrm{~S}$ in ethanol was made while stirred for $30 \mathrm{~min}$ at $60^{\circ} \mathrm{C}$ to obtain a milky homogeneous solution. Then, the solution was filled into a $100 \mathrm{~mL}$ Teflon lined stainless steel autoclave. The reaction was performed for $12 \mathrm{~h}$ at $220^{\circ} \mathrm{C}$. After cooling down to room temperature, the achieved black powder was washed with ethanol (twice) and dried in a vacuum oven at $80{ }^{\circ} \mathrm{C}$. In the next step, $0.5 \mathrm{~g}$ of obtained $\mathrm{MoS}_{2}$ powder was dispersed in $20 \mathrm{~mL}$ ethanol and stirred for $20 \mathrm{~min}$. Three suspensions individually were prepared and separately exposed under $532 \mathrm{~nm} \mathrm{Nd:YAG} \mathrm{pulsed} \mathrm{laser} \mathrm{irradiation} \mathrm{(} 5 \mathrm{~ns}$ pulse duration and $7 \mathrm{~mm}$ beam diameter) for $15 \mathrm{~min}$ with different energies 40,60, and $80 \mathrm{~mJ}$. After laser irradiation, three suspensions were dried in a vacuum oven at $40^{\circ} \mathrm{C}$. The analysis was applied to the resulted powder. Figure 1 shows a flow chart of the synthesis process. 


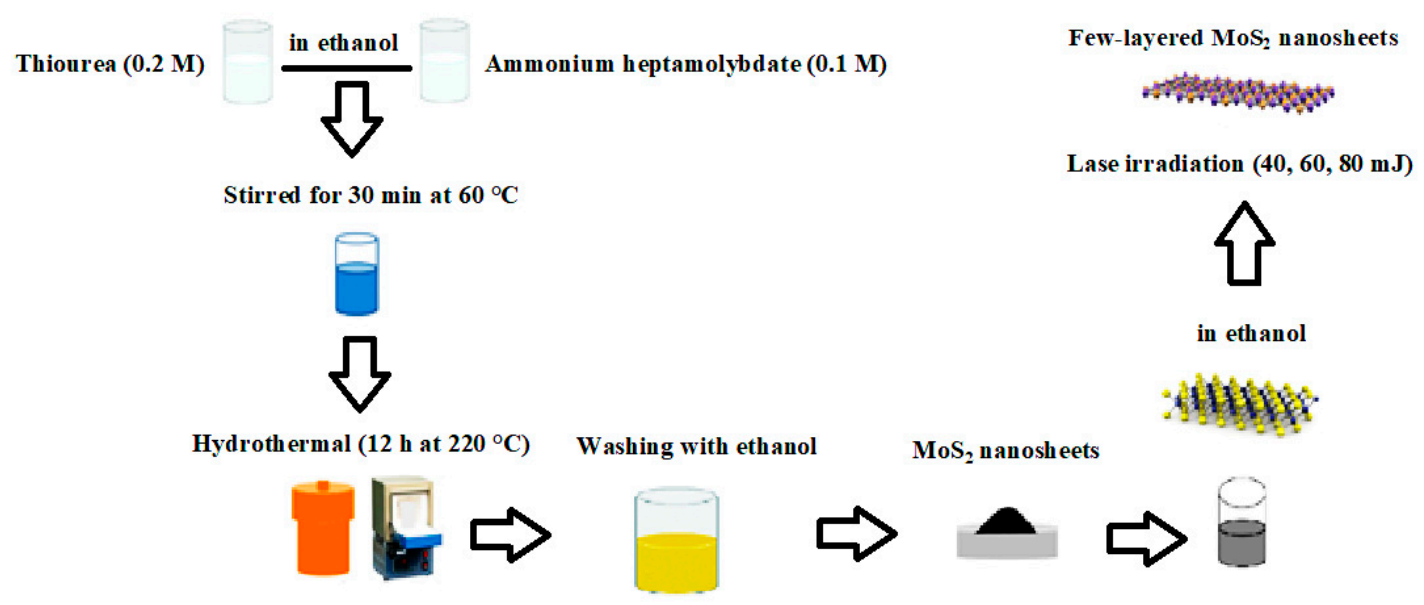

Figure 1. Flowchart of the synthesis process of the few-layered molybdenum disulfide $\left(\mathrm{MoS}_{2}\right)$ nanosheets.

\section{Characterization}

X-Ray diffraction (XRD) was utilized to study the structure of fabricated samples characterized by using X'Pert PRO (Malven Panalytical, Almelo (Netherlands)), with $\mathrm{Cu}-\mathrm{K} \alpha$ radiation, $(\lambda=0.154 \mathrm{~nm})$. Fourier transforms infrared (FTIR) spectra were applied using a spectrophotometer (FTIR-410) (Jasco, Toku, Japan), from 500 to $4000 \mathrm{~cm}^{-1}$. To determine the morphology and structure of the samples, transmission Electron microscopy (TEM) (Philips XL, Netherlands), was done. The bandgap of the samples calculated from UV-Vis optical absorption and transmission spectra (Lambda 750, PerkinElmer, Germany). The photoluminescence spectrum was recorded by a fluorescence spectrometer (PL, PerkinElmer LS 45, Germany).

\section{Results and Discussion}

The XRD of the $\mathrm{MoS}_{2}$ powder and few-layered $\mathrm{MoS}_{2}$ are shown in Figure 2. The existence peaks at $14.2^{\circ}, 32.6^{\circ}, 39.5^{\circ}, 44.2^{\circ}, 49.8^{\circ}$, and $58.3^{\circ}$ are consistent with the (002), (100), (103), (006), (105), and (110) planes of hexagonal $\mathrm{MoS}_{2}$ structure (JCPDS NO. 37-1492). By increasing laser energy, the intensity of these peaks is reduced. The calculated lattice constants $a=b=0.315 \mathrm{~nm}$, and $c=1.229 \mathrm{~nm}$ represent that all the samples have the crystallite nature of $\mathrm{MoS}_{2}$ materials. The (002) peak ascribing to a d-spacing of $0.62 \mathrm{~nm}$ illustrates the stacking of single layers, whereas peaks at higher angle are attributes of the crystallinity of the samples [37]. After laser irradiation, the intensity reduction of (002) peak proposed more discount in the thickness of $\mathrm{MoS}_{2}$ nanosheets [38,39]. It is considered that the (002) diffraction peak of the $\mathrm{MoS}_{2}$ powder, after laser irradiation, involves a shift to the lower angles and as a result, an increase in d-spacing indicating the destacking of $\mathrm{MoS}_{2}$ layers. It can be deduced with the enhancement in the laser energy the average grain sizes of the $\mathrm{MoS}_{2}$ nanosheets decrease. Therefore, the broadened diffraction peaks correspondingly confirm the decrease of the size of the $\mathrm{MoS}_{2}$ nanosheets, which is initiated by the laser irradiation.

The FTIR spectra of the samples are shown in Figure 3. The peaks below $700 \mathrm{~cm}^{-1}$ reveal the (Mo-O) deformation mode possibly caused by the sulfidation vibrations. The peaks between 750 and $1000 \mathrm{~cm}^{-1}$ indicate the most definite information on Mo-O coordination [40]. By increasing laser energy these peaks are more potent indicating stronger bonds of Mo-O. The absorption band between 1100 and $1650 \mathrm{~cm}^{-1}$ is ascribed to the stretching vibrations of the hydroxyl group and Mo-O vibrations. By reduction of $\mathrm{MoS}_{2}$ layers after laser irradiation, these peaks are stronger. The Mo-S vibration peak is around $600 \mathrm{~cm}^{-1}$, which is faded by increasing laser energy, meaning weaker bond of Mo-S. The C $=\mathrm{C}$ symmetric and asymmetric stretching vibrations are at 2890 and $2970 \mathrm{~cm}^{-1}$, which show small changes by different laser energies [41]. After laser irradiation, the peak at 1600 becomes stronger, which means stronger Mo-S bound, which is the result of monolayer $\mathrm{MoS}_{2}$ [42]. 


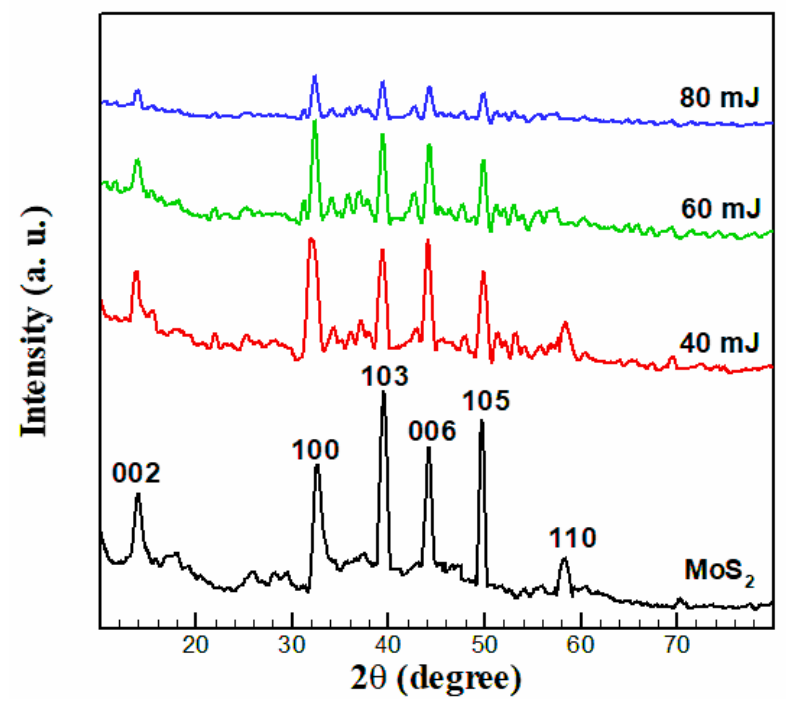

Figure 2. X-ray diffraction (XRD) pattern of the $\mathrm{MoS}_{2}$ structures under various laser energies from 40 to $80 \mathrm{~mJ}$.

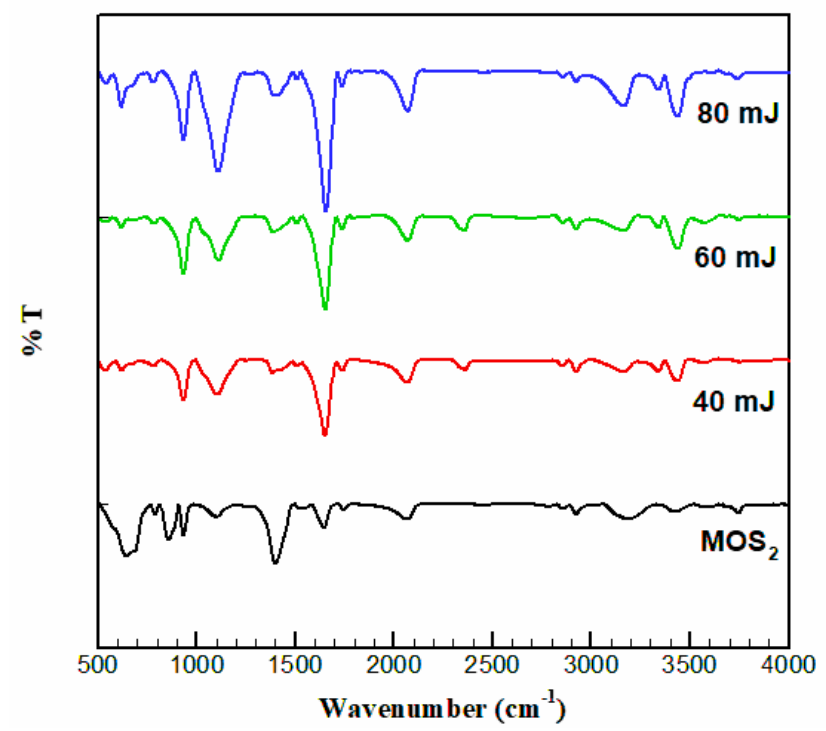

Figure 3. Fourier transform infrared spectroscopy (FTIR) spectrum of the $\mathrm{MoS}_{2}$ structures under various laser energies from 40 to $80 \mathrm{~mJ}$.

The TEM images of the $\mathrm{MoS}_{2}$ samples were performed to investigate the structural deviations that occur after various laser irradiation energies (Figure 4). Figure 4a is the image of $\mathrm{MoS}_{2}$ powder before laser irradiation. In Figure $4 \mathrm{~b}$, the large area could be distinguished for $40 \mathrm{~mJ}$ laser energy. By increasing laser irradiation energy, decreasing of the number of layers is obvious. Figure $4 \mathrm{c}$ shows the image after $60 \mathrm{~mJ}$ laser energy. Figure $4 \mathrm{~d}$ shows few-layered $\mathrm{MoS}_{2}$ nanosheets with folding at the edge, which is a phenomenon that occurs in two-dimensional materials. The number of layers could be determined using this morphological deviation [43]. A single dark line at the folded edge could be related to a monolayer [44]. The TEM results, in this study, indicate that the prepared $\mathrm{MoS}_{2}$ is few-layered with uniform size distribution. 


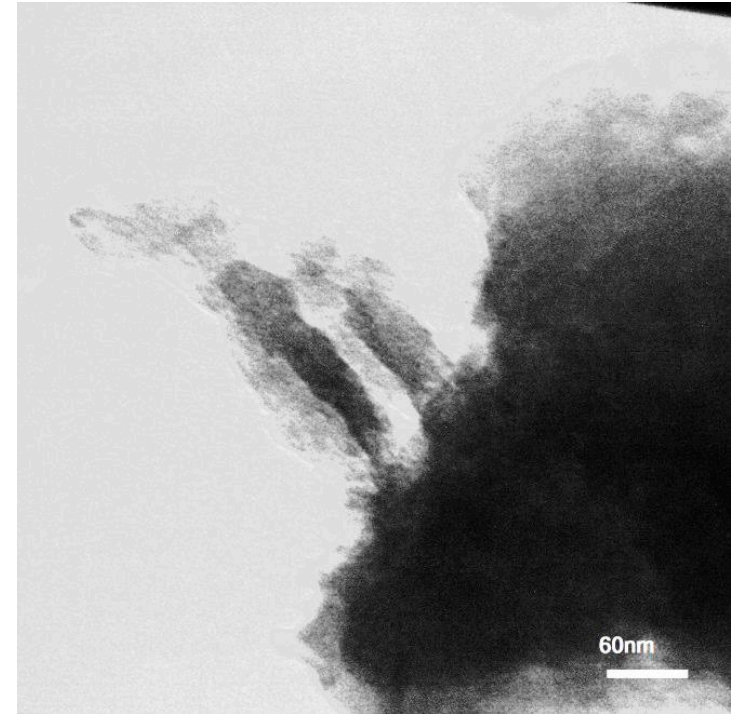

(a)

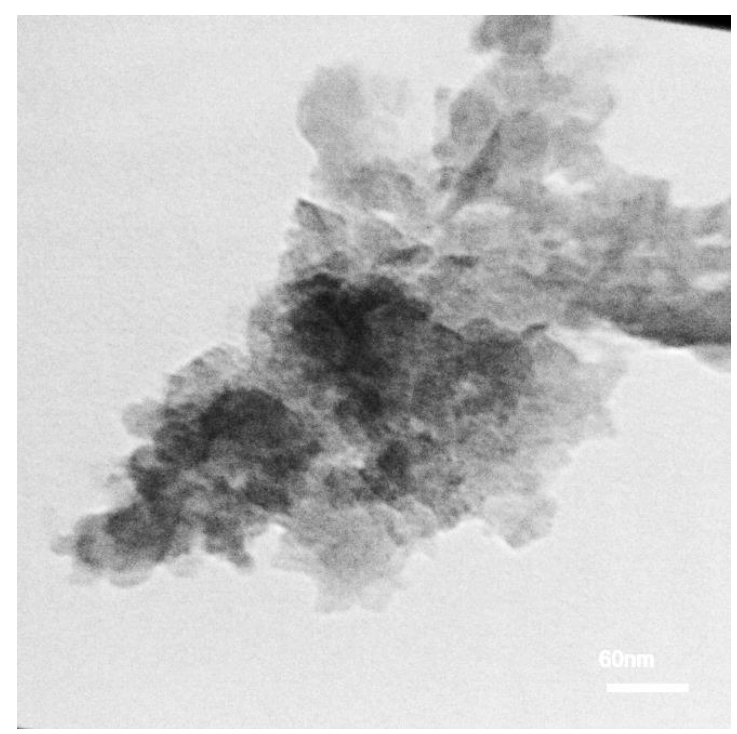

(c)

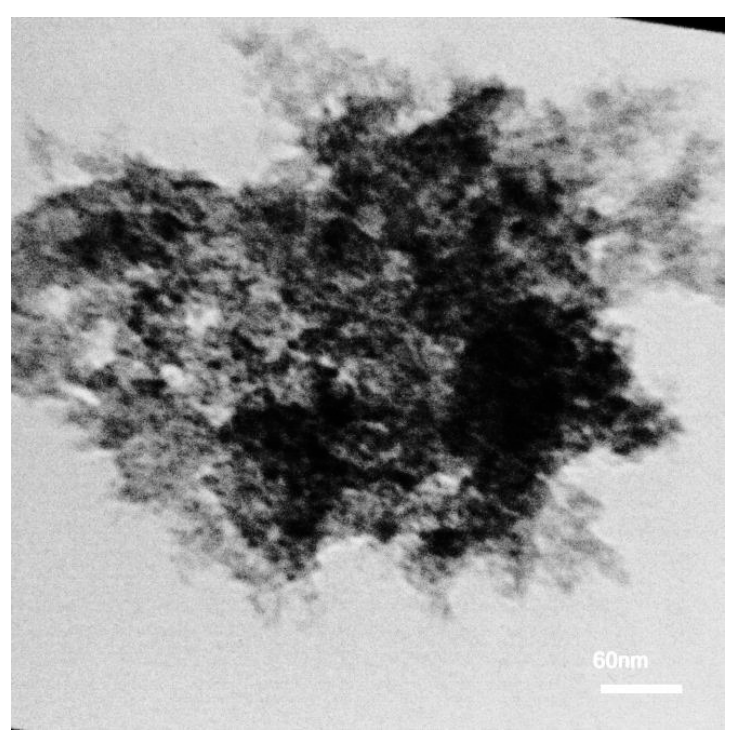

(b)

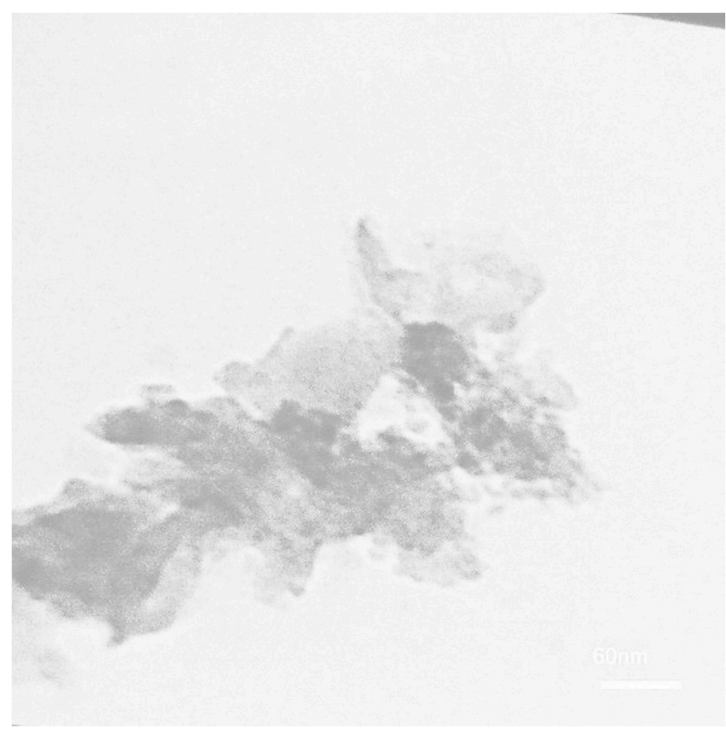

(d)

Figure 4. Transmission electron microscopy (TEM) images of (a) $\mathrm{MoS}_{2}$ powder, few-layered $\mathrm{MoS}_{2}$ nanosheets after (b) $40 \mathrm{~mJ}$; (c) $60 \mathrm{~mJ}$; and (d) $80 \mathrm{~mJ}$ laser energy (the scale for all images is $60 \mathrm{~nm}$ ).

To investigate the effect of laser energy on the absorption evaluation of $\mathrm{MoS}_{2}$, the few-layered $\mathrm{MoS}_{2}$ nanosheets were examined via UV-Vis spectroscopic technique by dispersing in water as a reference. Figure 5a shows the room temperature UV-Vis absorption spectra of the few-layered $\mathrm{MoS}_{2}$ nanosheets before laser irradiation and after $15 \mathrm{~min}$ laser irradiation under 40,60, and $80 \mathrm{~mJ}$ energies. The UV-Vis absorption spectrum of $\mathrm{MoS}_{2}$ solution displays a clear broad peak located at $250 \mathrm{~nm}$ ascribed to the excitonic property of $\mathrm{MoS}_{2}$ nanosheets [45]. It is different from the $2 \mathrm{D} \mathrm{MoS}$ counterpart with large lateral dimensions, which display four peaks at 340, 430, 590, and $650 \mathrm{~nm}$ as a characteristic absorption band of the original bulk $\mathrm{MoS}_{2}$. For bulk $\mathrm{MoS}_{2}$, the peaks at 590 and $650 \mathrm{~nm}$ are ascribed to the $\mathrm{K}$ point of the Brillouin zone, and the peaks at 340 and $430 \mathrm{~nm}$ are ascribed to the direct transition from the deep valence band to the conduction band [26,46]. Nonetheless, these four characteristic absorption bands fade in the spectra of the as-prepared $\mathrm{MoS}_{2}$ nanosheets. Only one peak is detected in the near-UV region $(\lambda<300 \mathrm{~nm})$ ascribed to the excitonic property of $\mathrm{MoS}_{2}$ [47]. The strong blue shift in the optical absorption is ascribed to the quantum confinement and edge effects $[47,48]$ upon the 
reduction of the lateral size of the $\mathrm{MoS}_{2}$ nanosheets to $<50 \mathrm{~nm}$. Thus, quantum confinement in layered d-electron materials such as $\mathrm{MoS}_{2}$ paves the way to engineer the electronic structure of matter at the nanoscale. As the TEM results show, most of the as-prepared $\mathrm{MoS}_{2}$ nanosheets are $\sim 30 \mathrm{~nm}$ and a strong blue shift is detected; this finding is in a good agreement with the results reported elsewhere $[49,50]$.

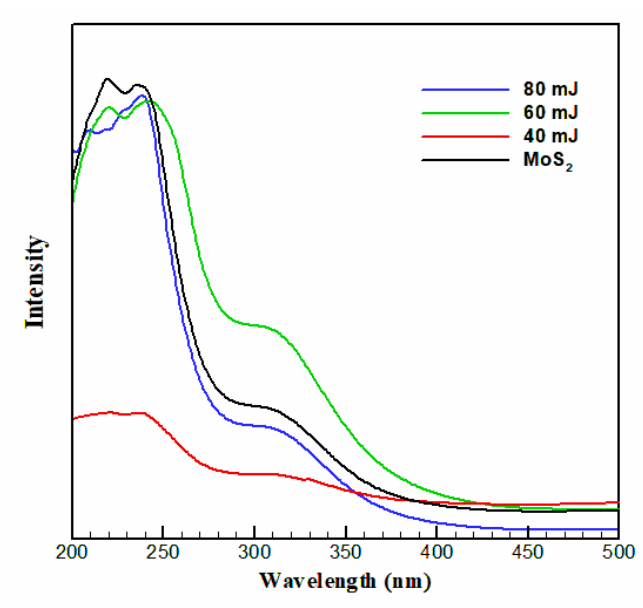

(a)

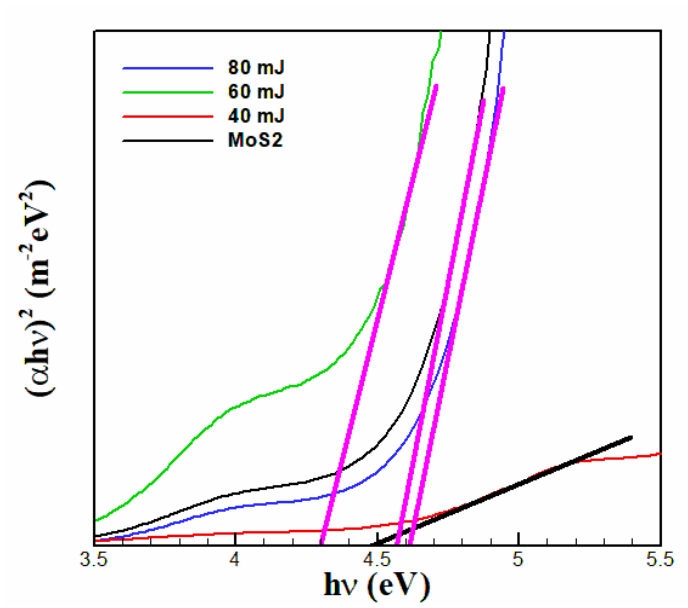

(b)

Figure 5. UV-Vis absorption spectrum of few-layered $\mathrm{MoS}_{2}$ nanosheets under various laser energies from 40 to $80 \mathrm{~mJ}$.

The absorption spectra of all four samples exhibit shoulders ranging from about $280 \mathrm{~nm}$ to $340 \mathrm{~nm}$ attributed to the interband transitions from the occupied $\mathrm{dz}^{2}$ orbital to unoccupied $\mathrm{dxy}, \mathrm{dyz}, \mathrm{dxz}$, and $d x^{2}-y^{2}$ orbitals [51]. From the UV-Vis data, it is possible to calculate the optical energy bandgap values of materials with a direct allowed bandgap by plotting the graph of $(\alpha \mathrm{h} v)^{2}$ versus $\mathrm{h} v$, according to the Tauc relation, $(\alpha \mathrm{h} v)^{n}=\mathrm{A}\left(\mathrm{h} v-\mathrm{E}_{\mathrm{g}}\right)$. In the Tauc equation, $\alpha$ is the absorption coefficient calculating from UV-Vis data according to the Beer-Lambert formula, $\mathrm{h} v$ is the photon energy in $\mathrm{eV}$, $\mathrm{E}_{\mathrm{g}}$ is the bandgap energy, and $\mathrm{A}$ is a constant. The symbol " $n$ " indicates the nature of the electronic transition causing the absorption and can have the values $2,1 / 2,2 / 3$, and $1 / 3$ if the electronic transition is direct allowed, indirect allowed, direct forbidden, and indirect forbidden transitions, respectively. The extrapolation of the linear part of the UV-Vis spectrum to the point in which $(\alpha \mathrm{h} v)^{2}=0$, gives the optical energy bandgap of the samples (Figure $5 b$ ). The calculated bandgap energies are greater than that of monolayer $\mathrm{MoS}_{2}(1.9 \mathrm{eV})$ and bulk $\mathrm{MoS}_{2}(1.2 \mathrm{eV})[26,52,53]$ and comparable to that of described monolayer $\mathrm{MoS}_{2}$ quantum dots (4.96 eV) [54]. The data are collected in Table 1. The slight difference in bandgap is ascribed to the size of nanosheets.

Conventional semiconductors like silicon have a bandgap in the range $1-1.5 \mathrm{eV}$, whereas wide bandgap (WBG) materials have bandgaps in the range $2-6 \mathrm{eV}$ such as $\mathrm{SiC}, \mathrm{GaN}$, and diamond $[55,56]$. Among them, gallium oxide $\left(\mathrm{Ga}_{2} \mathrm{O}_{3}\right)$ has the largest band gap $(\sim 4.66 \mathrm{eV})$ showing the widest tunable spectral range as compared with those of the other WBG semiconductors. The $\mathrm{MoS}_{2}$ nanosheets prepared in this study, are in the range of WBG semiconductors. Among the samples, the one under $80 \mathrm{~mJ}$ laser energy has the widest bandgap $(4.7 \mathrm{eV})$. Therefore, it is suitable to use in optoelectronic and electronic devices.

Table 1. Bandgap calculation from Figure 6 (error $0.05 \mathrm{eV}$ ).

\begin{tabular}{ccccc}
\hline Sample & $\mathbf{M o S}_{\mathbf{2}}$ & $\mathbf{4 0} \mathbf{~ \mathbf { J }}$ & $\mathbf{6 0} \mathbf{~ \mathbf { J }}$ & $\mathbf{8 0} \mathbf{~ m J}$ \\
\hline Bandgap (eV) & 4.6 & 4.5 & 4.3 & 4.7 \\
\hline
\end{tabular}




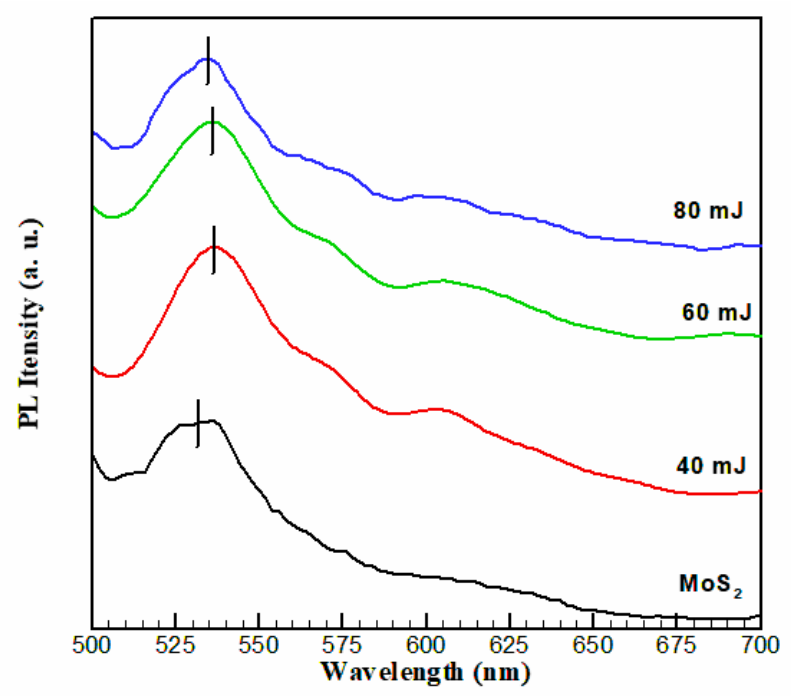

Figure 6. Room temperature photoluminescence spectra of the few-layered $\mathrm{MoS}_{2}$ under various laser energies from 40 to $80 \mathrm{~mJ}$.

The $\mathrm{MoS}_{2}$ nanostructure has specific photoluminescence capabilities due to the quantum size effect $[57,58]$. By thinning the $\mathrm{MoS}_{2}$ crystal to a monolayer, strong photoluminescence appears showing an indirect to direct bandgap transition in this d-electron system [59]. Figure 6 shows the PL spectrum of $\mathrm{MoS}_{2}$ nanosheets solution excited at $400 \mathrm{~nm}$. A strong sharp peak at $530 \mathrm{~nm}$ as a typical fluorescence spectrum of $\mathrm{MoS}_{2}$ particles with nanometer scale lateral dimensions is observed [60]. The PL peak at $530 \mathrm{~nm}$ can be attributed to $\mathrm{MoS}_{2}$ nanosheets with a lateral dimension up to a few tens of $\mathrm{nm}$ [61]. This is also supported by the TEM images showing the formation of few-layered $\mathrm{MoS}_{2}$ nanosheets. The PL spectrum is different from the two distinguished peaks positioned at around 610 and $661 \mathrm{~nm}$ in $2 \mathrm{D} \mathrm{MoS}_{2}$ flakes with large lateral dimensions from the direct bandgap of the K point [26]. It is remarkably hard to explain a shift in the PL of the samples because many factors such as strain [62] or quantum confinement [63] have an effect on the shift. This phenomenon could also be caused by the presence of several trap states [52,64]. It is noticeable, after laser irradiation, there is a redshift in the PL peak of the samples and its intensity decreases. This property is attributed to the existence of polydispersity of $\mathrm{MoS}_{2}$ nanosheets [46,65], the hot PL from Brillouin zone's K point, and many trap states in $\mathrm{MoS}_{2}$ nanosheets as detected in graphene quantum dots [65]. Reduction of the intensity means a reduction in electron-hole recombination.

The bandgap calculated by PL emission is always less than the original bandgap and is not the exact bandgap. The PL emission is mostly affected by the polarity of the solvent system and the changing solvent polarity causes a shift for the peaks. However, the absorption spectrum is much less affected by change in solvent polarity. Therefore, the bandgap is calculated from the absorbance spectrum. Bao et al. reported the change in PL peak position by the changing in excitation wavelength [66]. The PL spectra of $\mathrm{MoS}_{2}$ were measured at various excitation wavelengths. By increasing the excitation wavelength, the PL emission peaks shift to longer wavelengths. This phenomenon can also be due to the presence of several trap states, defects, and quantum confinements in layered material such as $\mathrm{MoS}_{2}$ [67].

Researches on semiconductors, especially WBG semiconductors, have fabricated various power devices, which are reliable and performant enough to design high-efficiency level converters to match application requirements. The WBG semiconductors are significant scientifically and technologically, and they have become the main materials to be applied in high-performance optoelectronic and electronic devices. The WBG semiconductors have many benefits for optoelectronic and electronic purposes because of their large bandgap energy and they help devices operate at much higher temperatures, voltages, and frequencies [68]. The power electronic modules make by these materials 
are considerably more powerful and energy efficient as compared with those made from conventional semiconductor materials [69]. They are becoming popular in high voltage and high power applications such as the traction motors and inverters found in the automotive sector [70]. The nature of WBG energy is appropriate for absorbing or emitting ultraviolet (UV) light in optoelectronic devices. For optical absorption and emission of the UV lights, WBG materials are significantly involved. The prepared WBG few-layered $\mathrm{MoS}_{2}$ nanosheets, in this study, are suitable for the mentioned applications.

\section{Conclusions}

A new, quick, green, and one-step method was introduced for preparing few-layered $\mathrm{MoS}_{2}$ nanosheets by applying the laser irradiation. Uniform size distribution and a high production rate of few-layered $\mathrm{MoS}_{2}$ nanosheets were achieved by solely adjusting the laser energy. Samples under 40, 60 , and $80 \mathrm{~mJ}$ laser energy were fabricated. The calculation displayed the widest bandgap, i.e., $4.7 \mathrm{eV}$, was for the sample under $80 \mathrm{~mJ}$ laser energy. The as-prepared $\mathrm{MoS}_{2}$ nanosheets exert strong quantum confinement and edge effects. $\mathrm{MoS}_{2}$ nanosheets are a type of WBG semiconductor having minor, quicker, more reliable power electronic components with high efficiency. Increasing the temperature reduces the bandgap of a semiconductor, thereby affecting most of the semiconductor material parameters. The spacing of the electronic levels and the bandgap increases with decreasing particle size, therefore, they are more compact in size and, as a result, less design margin is required to allow for temperature variation, allowing designers to achieve better performance from these devices. To the best of our knowledge, this is the first time to apply this method to exfoliate the $\mathrm{MoS}_{2}$ sheets.

Author Contributions: Conceptualization, S.K.; methodology, S.K. and M.M.; Validation, S.K. and M.M.; formal analysis, M.M.; investigation, S.K. and M.M.; Resources, M.M.; writing-original draft preparation, S.K. and F.A.; writing-review and editing, S.K. and F.A.; Visualization, S.K. and F.A.; supervision, S.K.; project administration, S.K. All authors have read and agreed to the published version of the manuscript.

Funding: This research received no external funding.

Conflicts of Interest: The authors declare no conflict of interest.

\section{References}

1. Néstor, P.; Elías, A.L.; Berkdemir, A.; Castro-Beltran, A.; Gutiérrez, H.R.; Feng, S.; Lv, R.; Hayashi, T.; López-Urías, F.; Ghosh, S.; et al. Photosensor device based on few-layered $\mathrm{WS}_{2}$ films. Adv. Funct. Mater. 2013, 23, 5511-5517.

2. Zhao, G.; Li, J.; Ren, X.; Chen, C.; Wang, X. Few-layered graphene oxide nanosheets as superior sorbents for heavy metal ion pollution management. Environ. Sci. Technol. 2011, 45, 10454-10462.

3. Jun, F.; Sun, X.; Wu, C.; Peng, L.; Lin, C.; Hu, S.; Yang, J.; Xie, Y. Metallic few-layered VS 2 ultrathin nanosheets: High two-dimensional conductivity for in-plane supercapacitors. J. Am. Chem. Soc. 2011, 133, 17832-17838.

4. Jafari, M.; Asadpour, M.; Majelan, N.A.; Faghihnasiri, M. Effect of boron and nitrogen doping on electro-optical properties of armchair and zigzag graphyne nanoribbons. Comput. Mater. Sci. 2014, 82, 391-398. [CrossRef]

5. Ke, Q.; Wang, J. Graphene-based materials for supercapacitor electrodes-A review. J. Mater. 2016, 2, 37-54.

6. Francesco, B.; Bartolotta, A.; Coleman, J.N.; Backes, C. 2D-crystal-based functional inks. Adv. Mater. 2016, 28, 6136-6166.

7. Bo, L.; Zhou, L.; Wu, D.; Peng, H.; Yan, K.; Zhou, Y.; Liu, Z. Photochemical chlorination of graphene. ACS Nano 2011, 5, 5957-5961.

8. Peng, G.; Song, H.; Chen, X. Electrochemical performance of graphene nanosheets as anode material for lithium-ion batteries. Electrochem. Commun. 2009, 11, 1320-1324.

9. Yuan, H.; Wu, J.; Xu, X.; Ho, Y.; Ni, G.; Zou, Q.; Koon, G.K.W.; Zhao, W.; Neto, A.H.C.; Eda, G.; et al. An innovative way of etching $\mathrm{MoS}_{2}$ : Characterization and mechanistic investigation. Nano Res. 2013, 6, 200-207.

10. Li, L.; Yu, Y.; Ye, G.J.; Ge, Q.; Ou, X.; Wu, H.; Feng, D.; Chen, X.H.; Zhang, Y. Black phosphorus field-effect transistors. Nat. Nanotechnol. 2014, 9, 372. 
11. Yu, Y.; Li, C.; Liu, Y.; Su, L.; Zhang, Y.; Cao, L. Controlled scalable synthesis of uniform, high-quality monolayer and few-layer $\mathrm{MoS}_{2}$ films. Sci. Rep. 2013, 3, 1866.

12. Novoselov Kostya, S.; Jiang, D.; Schedin, F.; Booth, T.J.; Khotkevich, V.V.; Morozov, S.V.; Geim, A.K. Two-dimensional atomic crystals. Proc. Nat. Acad. Sci. USA 2005, 102, 10451-10453. [CrossRef] [PubMed]

13. Coleman Jonathan, N.; Lotya, M.; O’Neill, A.; Bergin, S.D.; King, P.J.; Khan, U.; Young, K.; Gaucher, A.; De, S.; Smith, R.J.; et al. Two-dimensional nanosheets produced by liquid exfoliation of layered materials. Science 2011, 331, 568-571. [CrossRef] [PubMed]

14. Peter, M.; Khan, U.; Hughes, J.M.; Coleman, N.J. Role of solubility parameters in understanding the steric stabilization of exfoliated two-dimensional nanosheets by adsorbed polymers. J. Phys. Chem. C 2012, 116, 11393-11400.

15. Eswaraiah, V.; Backes, C.; Paton, K.R.; Harvey, A.; Gholamvand, Z.; McCauley, J.; Coleman, J.N. Large-scale production of size-controlled $\mathrm{MoS}_{2}$ nanosheets by shear exfoliation. Chem. Mater. 2015, 27, 1129-1139.

16. Liu, L.; Zeng, Z.; Cao, X.; Lu, G.; Wang, L.H.; Fan, Q.L.; Huang, W.; Zhang, H. Preparation of $\mathrm{MoS}_{2}$-polyvinylpyrrolidone nanocomposites for flexible nonvolatile rewritable memory devices with reduced graphene oxide electrodes. Small 2012, 8, 3517-3522.

17. Chouhan Raghuraj, S.; Žitko, G.; Fajon, V.; Živković, I.; Pavlin, M.; Berisha, S.; Jerman, I.; Vesel, A.; Horvat, M. A Unique Interactive Nanostructure Knitting based Passive Sampler Adsorbent for Monitoring of $\mathrm{Hg}_{2+}$ in Water. Sensors 2019, 19, 3432. [CrossRef]

18. Dong, Y.; Wang, Q.; Wu, H.; Chen, Y.; Lu, C.H.; Chi, Y.; Yang, H.H. Graphitic carbon nitride materials: Sensing, imaging and therapy. Small 2016, 12, 5376-5393.

19. Tian, J.; Liu, Q.; Asiri, A.M.; Qusti, A.H.; Al-Youbi, A.O.; Sun, X. Ultrathin graphitic carbon nitride nanosheets: A novel peroxidase mimetic, Fe doping-mediated catalytic performance enhancement and application to rapid, highly sensitive optical detection of glucose. Nanoscale 2013, 5, 11604-11609.

20. Manish, C.; Shin, H.S.; Eda, G.; Li, L.; Loh, K.P.; Zhang, H. The chemistry of two-dimensional layered transition metal dichalcogenide nanosheets. Nat. Chem. 2013, 5, 263.

21. Fai, M.K.; Shan, J. Photonics and optoelectronics of 2D semiconductor transition metal dichalcogenides. Nat. Photonics 2016, 10, 216.

22. Hua, W.Q.; Kalantar-Zadeh, K.; Kis, A.; Coleman, J.N.; Strano, M.S. Electronics and optoelectronics of two-dimensional transition metal dichalcogenides. Nat. Nanotechnol. 2012, 7, 699.

23. Shi, H.; Yan, R.; Bertolazzi, S.; Brivio, J.; Gao, B.; Kis, A.; Jena, D.; Xing, H.G.; Huang, L. Exciton dynamics in suspended monolayer and few-layer $\mathrm{MoS}_{2} 2 \mathrm{D}$ crystals. ACS Nano 2013, 7, 1072-1080.

24. Erwin, P.; Gelato, L.; Chabot, B.; Penzo, M.; Cenzual, K.; Gladyshevskii, R. TYPIX Standardized Data and Crystal Chemical Characterization of Inorganic Structure Types; Springer: Berlin/Heidelberg, Germany, 2013.

25. Lebegue, S.; Eriksson, O. Electronic structure of two-dimensional crystals from ab initio theory. Phys. Rev. B 2009, 79, 115409. [CrossRef]

26. Fai, M.K.; Lee, C.; Hone, J.; Shan, J.; Heinz, T.F. Atomically thin $\mathrm{MoS}_{2}$ : A new direct-gap semiconductor. Phys. Rev. Lett. 2010, 105, 136805.

27. Shi, Y.; Zhou, W.; Lu, A.; Fang, W.; Lee, Y.; Hsu, A.L.; Kim, S.M.; Kim, K.K.; Yang, H.Y.; Li, L.; et al. van der Waals epitaxy of $\mathrm{MoS}_{2}$ layers using graphene as growth templates. Nano Lett. 2012, 12, 2784-2791.

28. John, B.; Li, M.; Wang, S.; Wang, P.; Papakonstantinou, P. Electrocatalytic hydrogen evolution reaction on edges of a few layer molybdenum disulfide nanodots. ACS Appl. Mater. Interfaces 2015, 7, 14113-14122.

29. Jacob, B.; Moses, P.G.; Jaramillo, T.F.; Nørskov, J.K.; Chorkendorff, I. Hydrogen evolution on nano-particulate transition metal sulfides. Faraday Discuss. 2009, 140, 219-231.

30. Chen, L.; Zheng, X.; Du, Z.; Jia, B.; Gu, M.; Hong, M. A frozen matrix hybrid optical nonlinear system enhanced by a particle lens. Nanoscale 2015, 7, 14982-14988.

31. Tsai, M.L.; Su, S.H.; Chang, J.K.; Tsai, D.S.; Chen, C.H.; Wu, C.I.; Li, L.J.; Chen, L.J.; He, J.H. Monolayer MoS 2 heterojunction solar cells. ACS Nano 2014, 8, 8317-8322.

32. Mariyappan, S.; Bansal, T.; Durcan, C.A.; Yu, B. Molybdenum disulphide/titanium dioxide nanocomposite-poly 3-hexylthiophene bulk heterojunction solar cell. Appl. Phys. Lett. 2012, 100, 153901.

33. Ho, W.; Yu, J.C.; Lin, J.; Yu, J.; Li, P. Preparation and photocatalytic behavior of MoS2 and WS2 nanocluster sensitized $\mathrm{TiO}_{2}$. Langmuir 2004, 20, 5865-5869.

34. Benavente, E.; Ana, M.A.S.; Mendizábal, F.; González, G. Intercalation chemistry of molybdenum disulfide. Coord. Chem. Rev. 2002, 224, 87-109. [CrossRef] 
35. Lee, Y.S.; Zhang, X.Q.; Zhang, W.; Chang, M.T.; Lin, C.T.; Chang, K.D.; Yu, Y.C.; Wang, J.T.; Chang, C.S.; Li, L.J.; et al. Synthesis of large-area $\mathrm{MoS}_{2}$ atomic layers with chemical vapor deposition. Adv. Mater. 2012, $24,2320-2325$.

36. Xie, J.; Zhang, H.; Li, S.; Wang, R.; Sun, X.; Zhou, M.; Zhou, J.; Lou, X.W.; Xie, Y. Defect-rich MoS 2 ultrathin nanosheets with additional active edge sites for enhanced electrocatalytic hydrogen evolution. Adv. Mater. 2013, 25, 5807-5813.

37. Afanasiev, P. Synthetic approaches to the molybdenum sulfide materials. Comptes Rendus Chim. 2008, 11, 159-182. [CrossRef]

38. Bindhu, B.; Sharu, B.K.; Gopika, M.S.; Praseetha, P.K.; Veluraja, K. Molybdenum disulfide nanoflakes through Li-AHA assisted exfoliation in an aqueous medium. RSC Adv. 2016, 6, 22026-22033. [CrossRef]

39. Hung, P.V.; Kim, K.; Jung, D.; Singh, K.; Oh, E.; Chung, J.S. Liquid phase co-exfoliated MoS $_{2}-$ graphene composites as anode materials for lithium ion batteries. J. Power Sources 2013, 244, 280-286.

40. Kumar, K.; Santhana, A.; Jiang, S.; Warchoł, J.K. Synthesis and characterization of two-dimensional transition metal dichalcogenide magnetic $\mathrm{MoS}_{2} @ \mathrm{Fe}_{3} \mathrm{O}_{4}$ nanoparticles for adsorption of $\mathrm{Cr}$ (VI)/Cr (III). ACS Omega 2017, 2, 6187-6200. [CrossRef]

41. Khawula Tobile, N.Y.; Raju, K.; Franklyn, P.J.; Sigalas, I.; Ozoemena, K.I. The effects of morphology re-arrangements on the pseudocapacitive properties of mesoporous molybdenum disulfide $\left(\mathrm{MoS}_{2}\right)$ nanoflakes. J. Electrochem. Soc. 2016, 163, A1927-A1935. [CrossRef]

42. Tobias, K.; Heydrich, S.; Hirmer, M.; Schmutzler, J.; Schüller, C. Low-temperature photocarrier dynamics in monolayer $\mathrm{MoS}_{2}$. Appl. Phys. Lett. 2011, 99, 102109.

43. Meyer Jannik, C.; Geim, A.K.; Katsnelson, M.I.; Novoselov, K.S.; Booth, T.J.; Roth, S. The structure of suspended graphene sheets. Nature 2007, 446, 60. [CrossRef] [PubMed]

44. Sagar, P.; Harle, A.; Sathaye, S.; Patil, K. Development of a novel method to grow mono-/few-layered MoS 2 films and $\mathrm{MoS}_{2}$-Graphene hybrid films for supercapacitor applications. CrystEngComm 2014, 16, 10845-10855.

45. Dong, H.H.; Han, D.J.; Choi, J.S.; Park, M.; Seo, T.S. Dual role of blue luminescent MoS $_{2}$ quantum dots in fluorescence resonance energy transfer phenomenon. Small 2014, 10, 3858-3862.

46. Deepesh, G.; Damien, D.; Shaijumon, M.M. MoS2 quantum dot-interspersed exfoliated $\mathrm{MoS}_{2}$ nanosheets. ACS Nano 2014, 8, 5297-5303.

47. Chikan, V.; Kelley, D.F. Size-dependent spectroscopy of MoS2 nanoclusters. J. Phys. Chem. B 2002, 106, 3794-3804. [CrossRef]

48. Jaramillo Thomas, F.; Jørgensen, K.P.; Bonde, J.; Nielsen, J.H.; Horch, S.; Chorkendorff, I. Identification of active edge sites for electrochemical $\mathrm{H}_{2}$ evolution from $\mathrm{MoS}_{2}$ nanocatalysts. Science 2007, 317, 100-102. [CrossRef]

49. Xu, S.; Li, D.; Wu, P. One-pot, facile, and versatile synthesis of monolayer $\mathrm{MoS}_{2} / \mathrm{WS}_{2}$ quantum dots as bioimaging probes and efficient electrocatalysts for hydrogen evolution reaction. Adv. Funct. Mater. 2015, 25, $1127-1136$.

50. Dai, W.; Dong, H.; Fugetsu, B.; Cao, Y.; Lu, H.; Ma, X.; Zhang, X. Tunable fabrication of molybdenum disulfide quantum dots for intracellular microRNA detection and multiphoton bioimaging. Small 2015, 11, 4158-4164.

51. Bojana, V.; Dominko, R.; Gunde, M.K.; Hauptman, N.; Skapin, S.D.; Remskar, M. Optical properties of exfoliated $\mathrm{MoS}_{2}$ coaxial nanotubes-analogues of graphene. Nanoscale Res. Lett. 2011, 6, 593.

52. Václav, Š.; Henych, J. Strongly luminescent monolayered $\mathrm{MoS}_{2}$ prepared by effective ultrasound exfoliation. Nanoscale 2013, 5, 3387-3394.

53. Hao, H.; Du, C.; Shi, H.; Feng, X.; Li, J.; Tan, Y.; Song, W. Water-Soluble Monolayer Molybdenum Disulfide Quantum Dots with Upconversion Fluorescence. Part. Part. Syst. Charact. 2015, 32, 72-79.

54. Ren, X.; Pang, L.; Zhang, Y.; Ren, X.; Fan, H.; Liu, S.F. One-step hydrothermal synthesis of monolayer $\mathrm{MoS}_{2}$ quantum dots for highly efficient electrocatalytic hydrogen evolution. J. Mater. Chem. A 2015, 3, 10693-10697.

55. Prestopino, G.; Marinelli, M.; Milani, E.; Verona, C.; Verona-Rinati, G. Transient lateral photovoltaic effect in synthetic single crystal diamond. Appl. Phys. Lett. 2017, 111, 143504. [CrossRef]

56. Eva, M.; Omnès, F.; Calle, F. Wide-bandgap semiconductor ultraviolet photodetectors. Semicond. Sci. Technol. 2003, 18, R33. 
57. Andrea, S.; Sun, L.; Zhang, Y.; Li, T.; Kim, J.; Chim, C.; Galli, G.; Wang, F. Emerging photoluminescence in monolayer $\mathrm{MoS}_{2}$. Nano Lett. 2010, 10, 1271-1275.

58. Yafei, L.; Zhou, Z.; Zhang, S.; Chen, Z. $\mathrm{MoS}_{2}$ nanoribbons: High stability and unusual electronic and magnetic properties. J. Am. Chem. Soc. 2008, 130, 16739-16744.

59. Wei, L.; Birdwell, A.G.; Amani, M.; Burke, R.A.; Ling, X.; Lee, Y.; Liang, X.; Peng, L.; Richter, C.A.; Kong, J.; et al. Broadband optical properties of large-area monolayer CVD molybdenum disulfide. Phys. Rev. B 2014, 90, 195434.

60. Subhrajit, M.; Maiti, R.; Midya, A.; Das, S.; Ray, S.K. Tunable direct bandgap optical transitions in $\mathrm{MoS}_{2}$ nanocrystals for photonic devices. ACS Photonics 2015, 2, 760-768.

61. Wu, J.Y.; Zhang, X.; Ma, X.; Qiu, Y.; Zhang, T. High quantum-yield luminescent $\mathrm{MoS}_{2}$ quantum dots with variable light emission created via direct ultrasonic exfoliation of $\mathrm{MoS}_{2}$ nanosheets. RSC Adv. 2015, 5, 95178-95182.

62. Shi, H.; Pan, H.; Zhang, Y.; Yakobson, B.I. Quasiparticle band structures and optical properties of strained monolayer $\mathrm{MoS}_{2}$ and $\mathrm{WS}_{2}$. Phys. Rev. B 2013, 87, 155304 .

63. Miller, D.A.B.; Chemla, D.S.; Schmitt-Rink, S. Relation between electroabsorption in bulk semiconductors and in quantum wells: The quantum-confined Franz-Keldysh effect. Phys. Rev. B 1986, 33, 6976. [CrossRef] [PubMed]

64. Shinde Dhanraj, B.; Vijayamohanan, K.P. Electrochemical preparation of luminescent graphene quantum dots from multiwalled carbon nanotubes. Chem. Eur. J. 2012, 18, 12522-12528. [CrossRef]

65. Deepesh, G.; Damien, D.; Li, B.; Gullappalli, H.; Pillai, V.K.; Ajayan, P.M.; Shaijumon, M.M. Electrochemical synthesis of luminescent $\mathrm{MoS}_{2}$ quantum dots. Chem. Commun. 2015, 51, 6293-6296.

66. Bao, L.; Zhang, Z.; Tian, Z.; Zhang, L.; Liu, C.; Lin, Y.; Qi, B.; Pang, D. Electrochemical tuning of luminescent carbon nanodots: From preparation to luminescence mechanism. Adv. Mater. 2011, 23, 5801-5806. [CrossRef]

67. Bo, L.; Li, X.; Ran, P.; Zuo, P.; Wang, A.; Qu, L.; Zhao, Y.; Cheng, Z.; Lu, Y. Preparation of monolayer MoS quantum dots using temporally shaped femtosecond laser ablation of bulk $\mathrm{MoS}_{2}$ targets in water. Sci. Rep. 2017, 7, 1-12.

68. Ahmed, E.; Chow, T.P. Silicon carbide benefits and advantages for power electronics circuits and systems. Proc. IEEE 2002, 90, 969-986.

69. Burak, O.; Tolbert, L.M. Comparison of Wide-Bandgap Semiconductors for Power Electronics Applications; Department of Energy: Washington, DC, USA, 2004. [CrossRef]

70. John, R.; Dorn-Gomba, L.; Mak, C.; Emadi, A. Automotive traction inverters: Current status and future trends. IEEE Trans. Veh. Technol. 2019, 68, 3337-3350.

(C) 2020 by the authors. Licensee MDPI, Basel, Switzerland. This article is an open access article distributed under the terms and conditions of the Creative Commons Attribution (CC BY) license (http://creativecommons.org/licenses/by/4.0/). 